\title{
LA MOTIVACIÓN DE LOGROS Y EL AUTO CONCEPTO EN ESTUDIANTES DE LA UNIVERSIDAD NACIONAL MAYOR DE SAN MARCOS
}

\author{
THE MOTIVATION OF PROFITS AND THE SELF CONCEPT IN STUDENTS OF THE \\ GREATER NATIONAL UNIVERSITY OF SAN MARCOS
}

\author{
LUIS VICUÑA PERI ${ }^{1}$ HECTOR HERNÁNDEZ VALZ, JOSÉ RIOS DIAZ \\ Universidad Nacional Mayor de San Marcos, Facultad de Psicología
}

\section{RESUMEN}

OBJETIVO: Identificar la relación entre el autoconcepto con los tipos de motivación propuestos por Mc Clelland: poder, afiliación y logro. Así mismo averiguar el papel que cumple el rendimiento académico y el sexo sobre la autoestima. Identificar el papel que cumple el rendimiento académico y el sexo sobre los componentes de la motivación: afiliación, poder y logro. DISEÑO: La presente investigación es correlacional y descriptiva. MATERIAL Y MÉTODOS: Trabajándose con una muestra de 768 estudiantes con especialidades académicas de áreas A, B y C. A ellos se les aplicó el cuestionario de autoconcepto de José Navas y el inventario de Motivación elaborados y revisado psicométricamente para este estudio. RESULTADOS: El análisis de resultados fue efectuado en 3 pasos. El primero mediante la correlación del autoconcepto con cada uno de los componentes de la motivación y de estos entre sí. El segundo paso fue mediante la contrastación del autoconcepto y de la motivación de afiliación o de logro en función al área académica y al sexo. Finalmente, se hizo una descripción de la muestra examinada en base a los promedios obtenidos y distribución porcentual por categorías. CONCLUSIONES: Se concluye que el autoconcepto con la motivación de filiación, poder y logro son variables independientes. Los componentes de la motivación correlacionan significativamente sobre ellos, lo que indica que son componentes de un mismo factor. Por otro lado, el autoconcepto tiende a ser influenciado por el sexo, y no así por el área académica de procedencia. En cambio, la motivación de afiliación es más afectada por el área académica que por el sexo; y tanto en la motivación de poder y de logro el sexo o el área de pertenencia académica influyen significativamente. Por los resultados descriptivos encontramos que más del $60 \%$ de la muestra examinada se ubica en las categorías de tendencia alta, lo que es un buen indicador respecto a la forma como se describen a sí mismos, como también en el motivo que puede mantenerles constantes en la búsqueda del éxito.

Palabras claves: Autoconcepto, motivación, motivación de logro.

\section{ABSTRACT}

OBJECTIVE: To identify the relation between autoconcepto with the types of motivation proposed by Mc Clelland: to be able, affiliation and profit. Also to find out the paper that fulfHls the academic yield and sex on the selfsteem. To identify the paper that fulfills the academic yield and sex on the components of the motivation: affiliation, power and I obtain. DESIGN: The present investigation is corelational and descriptive. MATERIAL And METHODS: Working with a sample of 768 students, with academic specialties of areas To, B and C. To them it was psychometrically applied to the questionnaire of self concept of Jose Navas and the elaborated and reviewed inventory to them of Motivation 
for this study. RESULTS: The analysis of results was carried out in 3 steps. First by means of the correlation of self concept with each one of the components of the motivation and these to each other. The second step was by means of the contrast of autoconcepto and the motivation of affiliation or profit in function to the academic area and sexo Finally, one became a description of the sample examined on the basis of the obtained averages and percentage distribution by categories. CONCLUSIONS: One concludes that self concept with the motivation of connection, power and profit is variable independent. The components of the motivation correlate significantly on them, which indicates that they are component of a same factor. On the other hand, self concept tends to be influenced by sex, and not thus by the academic area of origin However, the affiliation motivation more is affected by the academic area that by sex; and as much in the motivation of being able and profit the sex or the area of academic property they influence significantly. By the descriptive results we found that more of $60 \%$ of the examined sample it is located in the categories of high tendency, which is a good indicator with respect to the form as they are described themselves, like also in the reason that can maintain to them constant in the search of the success.

Keywords: Self concept, motivation, motivation of profit.

\section{INTRODUCCIÓN}

El concepto que cada persona tiene de sí mismo le sirve para enfrentarse a sí mismo, ante los demás, ante la vida y frente a diversas circunstancias con un determinado grado de éxito. Desde este punto de vista el estudiante universitario que en el común de los casos se encuentra de tránsito entre el periodo de la adolescencia hacia la juventud, periodos donde la exploración de las actitudes e intereses del primer periodo, le demandan en el siguiente, la afirmación en un contexto ocupacional, donde las aptitudes deben concentrarse hacia un campo determinado de interés, que en el mundo de las profesiones equivale a optar por una especialidad. Consideramos que esta compleja vivencia de la juventud universitaria, será favorable en la medida que su autoconcepto sea adecuado; por otro lado definida la elección queda aún por observar, el impulsor que asegure que el joven priorice a las actividades y tecnológicas de las actividades recreativas y la búsqueda del trabajo.

El joven sabe que continuando sus estudios podrá alcanzar lo segundo; pero luego de permanecer no menos de 6 años integrando y compartiendo la mayor parte de su tiempo en las aulas universitarias, este impulsor lo denominamos motivación, distinguiendo entre sus componentes la afiliación, el poder y el logro.

Si el joven tiene un adecuado concepto y la motivación requerida para mantenerse firme y constante en la consecución de sus metas, podemos prever éxito, donde la productividad será dependiente de lo que el sujeto ha logrado en la universidad y el impulsor para continuar en la conquista de sus metas. Bajo esta perspectiva, en el presente estudio universitario de alumnos de ambos sexos perteneciente a las tres áreas académicas de la UNMSM, nos proponemos despejar las siguientes interrogantes:

1. ¿Se relaciona el autoconcepto con la motivación de afiliación, poder y logro?

2. ¿Influye al área académica de pertenencia y el sexo sobre la autoestima?

3. ¿Influye el área académica de pertenencia y el sexo sobre los componentes de afiliación, poder y logro de la motivación? 
De las interrogantes formuladas se desprende que los objetivos centrales, de la presente investigación consisten: en identificar la relación entre el autoconcepto con los tipos de motivación propuestos por Mc Clelland5: poder, afiliación y logro. Así mismo averiguar el papel que cumple el rendimiento académico y el sexo sobre la autoestima. Identificar el papel que cumple el rendimiento académico y el sexo sobre los componentes de la motivación: afiliación, poder y logro.

La importancia de la presente investigación radica en averiguar si el autoconcepto se relaciona con la motivación, con la perspectiva de determinar si ambas variables son interdependientes para predecir la actividad productiva, colaborando así con la resolución de un problema teórico y un problema empírico, cotidiano en el proceso de enseñanza aprendizaje.

\section{Marco Teórico}

El autoconcepto es un proceso psicológico que permite al sujeto generalizar su modo de reaccionar ante las situaciones que se le presentan. William James, Erick Erickson, Sigmund Freud entre otros, citados en Ellis, A. (1994) ${ }^{1}$, consideran que el autoconcepto se constituye a partir de las tempranas experiencias del niño, en relación con la consecución de sus expectativas y la aprobación-desaprobación de las personas que tienen especial significado, primero los padres y parientes cercanos; luego los amigos, y finalmente, las múltiples interacciones sociales. Para estos investigadores, el autoconcepto controlado en una primera instancia pro el mundo externo, significativo para el niño, se internaliza, pasando de la idea de uno a partir de los otros, hacia la idea de uno, a partir del significado personal.

Desde el punto de vista de la teoría cognoscitiva, Markus Narius, en 1984, en Mahoney, M. $(1988)^{2}$ considera que el autoconcepto es el autoesquema que organiza y dirige el proceso de información a cerca de sí mismo.

De este modo, el autoconcepto se refiere al significado que el sujeto tiene acerca de sí mismo, acerca de su relación con los demás, ante situaciones nuevas, ante la vida y ante la aceptación que tiene para los demás. Para esta investigación considero conveniente enfatizar en la idea de significado, ya que es la opinión o juicio que el sujeto se atribuye, el autoconcepto responde al componente evaluativo de la personalidad; es, en todo caso, lo que el sujeto piensa acerca de sí mismo.

El autoconcepto de sí mismo es el significado que el sujeto se atribuye en función de sus características físicas y sus aptitudes motoras e intelectuales.

El autoconcepto, en relación con los demás es el significado que el sujeto atribuye a sus interacciones. El autoconcepto ante situaciones nuevas es el significado que el sujeto atribuye a su capacidad para manejar y afrontar situaciones imprevistas.

El autoconcepto ante la vida es la evaluación que el sujeto hace de sus circunstancias vitales de manera global.

Puntos de vista que en alguna medida también se enfatizan por Kuch, J. (1994)3 


\section{La motivación}

Madsen, K.B. (1977)4 Viene a ser el proceso de activación selectiva, que orienta la acción del individuo hacia la satisfacción de necesidades. La motivación es un fenómeno complejo y sutil. Una persona se comporta en respuesta a estímulos asociados con un estado hipotético de desequilibrio. La conducta se dirige a alcanzar un inventivo o meta que el individuo anticipa que será satisfactorio, que restablecerá el estado de equilibrio. Alcanzar la meta conduce a un cambio en el grado de desequilibrio y a un cambio concomitante en nivel de la fuerza que impulsa al individuo hacia la acción. Los estados internos de desequilibrio no pueden observarse directamente; su presencia y su naturaleza deben inferirse de la observación de las clases de conducta que se suceden en relación con diferentes tipos de incentivos y circunstancias antecedentes.

\section{La Teoría de la Motivación de Logro}

Al interior de la teoría de Mc. Clelland (1953), existen tres importantes tipos de motivación en el individuo. Aunque es probable encontrar cierto nivel de los tres, los individuos dirigen su comportamiento anteponiendo uno de ellos, aunque no son contradictorios. Estos tipos de motivación vienen a ser los siguientes:

\section{Motivos de Afiliación}

El deseo de la gente de establecer y mantener contactos interpersonales es un fuerte impulso que la mayoría de las personas presentan en cierto grado. Sin embargo, cuando este motivo es el que dirige y domina la acción de un individuo, vale decir, su necesidad de seguir perteneciendo promueve su especial manera de actuar, estamos frente a una motivación de afiliación. La afiliación implica en la mayoría de los casos un determinado nivel de dependencia frente a las decisiones que toma el grupo colocando además en una segunda posición lo que el individuo opina. Shachter, en Peyró (1984) ${ }^{6}$ sugiere que una razón importante de que la gente se reúna, se coalicione y forme grupos referenciales, es compartir sus opiniones con individuos de pensamiento similar. En cierto sentido, los grupos se forman a menudo para que los individuos ganen seguridad al oír que sus creencias son confirmadas por los otros, pero su dependencia ante el mismo los vuelve carentes de iniciativa y parametra sus expectativas. Por otro lado, la ansiedad puede ser otra razón importante se congregue. El motivo de la afiliación puede surgir como un tipo particular de motivación orientada a la seguridad, y por lo tanto no vendrían a ser una respuesta a la ansiedad, y no un estimulo positivo de goce.

\section{Motivos de Logro}

Relacionados con los deseos de estimación o competencia está en el deseo más general de lograr algo de importancia. Este tipo de impulso está referido al grado de satisfacción que deriva de lo que la gente realiza. Algunos parecen esforzarse mucho por obtener la sensación de haber aIcanzado una meta difícil; otros en cambio, parecen necesitar constante estimulación en la forma de recompensas tangibles. No sólo McClelland, citado en Brows Rogers $(1974)^{7}$ es quien propone este tipo de motivación; en esta concepción se encontraron trabajando desde la década de los 70 investigadores y teóricos de la talla de Atkinson, Clark, Lowell y otros, en Brows Rogers (1974) ${ }^{8}$. Para las personas con altos motivos de logro, la consumación resultante del proceso de esforzarse o procurar el logro es suficiente recompensa, por sí misma, para que el esfuerzo total sea significativo y valioso. Sin embargo, lo que puede constituir logro para unos, es probable que no lo sea para otros. La elección de lo que constituye logro, al menos en parte, depende de lo que el individuo puede realizar. Una persona con alta motivación de logro se esfuerza por tareas que le 
parecen importantes (juicio subjetivo), pero que a su vez considera alcanzables. Tenderá a desconocer las metas fáciles, porque su alcance no daría sensación real de consumación es demasiado baja.

Me. Clelland ${ }^{5}$ ha sostenido que el bienestar económico de una sociedad depende de la actividad empresarial $\mathrm{y}$ ha demostrado que esa actividad ha estado históricamente relacionada con la motivación de logro de sus miembros.

\section{Motivos de Poder}

Cuando la realización de la tarea se convierte en un instrumento para obtener algo, diferente a la aprobación social (donde se estaría observando un caso de motivación de afiliación) nos encontramos frente a la motivación de poder.

La búsqueda del poder, según la teoría de Me. Clelland ${ }^{5}$ se fundamenta en la necesidad del individuo por colocarse sobre los demás y ejercer sobre ellos algún tipo de controlo dominio. Cuando la actividad desempeñada con esmero tiene por finalidad colocar al actor en una mejor posición frente al grupo, o cuando intenta figurar como el mejor dotado para un ascenso, nos encontramos frente a la motivación por el poder.

Es importante señalar que aunque el desempeño de una persona con cualquiera de los tres tipos de motivación puede ser similar, la razón por la que lo hacen genera una diferencia importante. Alguien podría trabajar muy bien por la presión del grupo, por el interés que le despierta la tarea y el placer de desarrollarla a cabalidad, o por obtener algo más.

Se sustentan las siguientes hipótesis:

h1 El autoconcepto se relaciona significativamente con los componentes de afiliación, poder y logro de la motivación.

h2 El sexo influye significativamente sobre el autoconcepto

h3 El área de pertenencia académica influye significativamente sobre el autoconcepto.

h4 El sexo influye significativamente sobre los componentes de afiliación, logro y poder de la motivación.

h5 El área de pertenencia académica influye significativamente sobre la afiliación, logro y poder de la motivación.

Las variables estudiadas en la presente investigación son las siguientes:

Variable Independiente

El sexo y el área de pertenencia académica

Variable Dependiente

El autoconcepto y los componentes de afiliación, logro y poder de la motivación

Variable Controlada

Estudiantes de la Universidad Nacional Mayor de San Marcos.

\section{MATERIALES y MÉTODOS}

La presente investigación es correlacional y descriptiva

Muestra

Está constituida por 768 estudiantes, teniendo como base para la determinación muestral el tamaño de elementos requeridos para que cada item tenga una probabilidad de elección del 0.55 al 0.50 trabajando con un nivel de confianza del 0.05 de margen de error.

La distribución muestral fue la siguiente: 
El área académica A, constituida por 6 facultades representa al 33\% de las mismas, cuya cuota proporcional a la muestra viene a ser de 254 sujetos.

El área académica B, constituida por 8 facultades representa al $44 \%$ de las mismas, cuya cuota proporcional a la muestra viene a ser de 338 sujetos.

$\mathrm{El}$ área académica $\mathrm{C}$, constituida por 4 facultades representa al $22 \%$ de las mismas, cuya cuota proporcional a la muestra viene a ser de 176 sujetos.

Descripción de Instrumentos

Se utilizó el Inventario de Autoconcepto de José Navas, que evalúa cinco componentes: autoconcepto en relación a los demás, autoconcepto hacia sí mismo, autoconcepto hacia situaciones nuevas, autoconcepto hacia la vida y autoconcepto en relación a la aceptación de los demás.

Para evaluación de la motivación de afiliación, poder y logro se elaboró la escala denominada M-L-1996, constituida por 18 situaciones estructuradas y tres preguntas correspondientes a cada una, que hace un total de 54 items, La validez es por constructo, encontrándose correlaciones significativas entre cada uno de los componentes motivacionales que evalúa. La confiabilidad se realizó por el método del test reset, encontrándose un coeficiente de 0.88 al aplicarse la prueba con un intervalo de 03 meses desde su utilización inicial.

Técnicas de Recolección de Información

La aplicación de ambas pruebas se efectuó en grupos no mayores de 25 estudiantes por primera vez, mostrando que no todos los evaluados requeridos mostraron su interés por participar.

Método de Procesamiento de Datos

Se trabajó calculando la correlación del autoconcepto con cada uno de los componentes, utilizando el análisis producto de momentos de Pearson y la prueba t de significación de las mismas. Para contrastar los resultados fueron sometidos al análisis de varianza para dos factores: área de pertenencia académica y sexo.

\section{RESULTADOS}

El análisis tiene tres momentos; el primero corresponde a las correlaciones entre el autoconcepto con los componentes de la motivación, el segundo a la constrastación del autoconcepto y los componentes motivacionales en función al sexo y área académica de pertenencia. El tercer análisis es descriptivo, en función a los resultados.

Relación entre el autoconcepto con los componentes motivacionales

Para establecer si el autoconcepto se relaciona con los componentes de afiliación, poder y logro de la motivación, y de estos entre ·sí mismos, se procedió a estimar los coeficientes de correlación, cuyos resultados se reproducen en el siguiente cuadro:

\section{Cuadro $\mathrm{N}^{\circ} 1$ De Coeficientes de Correlación del Autoconcepto con los Componentes Motivacionales y de estos entre ellos.}

\begin{tabular}{|l|l|l|l|}
\hline & Afiliación & Poder & Logro \\
\hline Autoconcepto & 0.14 & -0.04 & 0.011 \\
Afiliación & & 0.48 & 0.40 \\
Poder & & & 0.73 \\
\hline
\end{tabular}


Del cuadro se desprende que el autoconcepto no correlaciona con los componentes de la motivación, lo que significa que la autodescripción que una persona se hace de sí mismo no determina a los impulsores para concretar acciones específicas.

La correlación más baja encontrada en los componentes motivacionales es de filiación con logro, que es igual a 0.040 , que tiene un valor $\mathrm{t}$ de12.08 que resulta muy significativa, aún para el 0.01 de margen de error. Ello significa que todos los componentes son partes de un mismo factor, que viene a ser la motivación.

El autoconcepto y los componentes motivacionales de afiliación, poder y logro en función al sexo y área académica de pertenencia. Para este análisis los resultados encontrados del autoconcepto y de cada uno de los componentes motivaciones fueron sometidos al análisis de varianza para dos factores, habiéndose encontrado:

\section{a. El autoconcepto}

Al efectuar la contrastación, teniendo en cuenta al total de la muestra exami!1ada encontramos una razón $F$ de 3,23 que al 0.05 de riesgo de error, la $F^{\prime}$ es igual al. 71. Como se puede observar la diferencia es significativa, lo que muestra que el autoconcepto está sujeto a variación.

Al analizar dicha variación, pero en función al sexo encontramos una razón $\mathrm{F}$ de 3.52, siendo la $F^{\prime}$ al 0.05 de 3.84. La diferencia no es significativa a este margen de error. Sin embargo, se puede observar que el sexo plantea tendencias hacia la diferencia.

Respecto al área de pertenencia académica, la razón $\mathrm{F}$ es de 0.94 , siendo al $\mathrm{F}^{\prime}$ al 0.05 igual a 3. Ello indica que esta variable no tiene el menor efecto. Con este resultado es necesario calcular la interacción entre la variable sexo y el área de pertenencia, encontrando una razón $F$ de 19.89, que para una $F^{\prime}$ igual a 3 es muy significativa. Este dato ratifica que la diferencia encontrada en todo el grupo se debe a la influencia del sexo sobre el autoconcepto.

\section{b. La motivación de afiliación}

En un análisis similar al anterior encontramos una razón $\mathrm{F}$ para todo el grupo igual a 1.82, que resulta significativa al 0.05 de margen de error $\left(\mathrm{F}^{\prime}\right.$ al $\left.0.05=1.71\right)$

Por este dato, el análisis en función al sexo arroja una razón $\mathrm{F}$ del 0.004 , que resulta despreciable.

En función al área de pertenencia la razón $\mathrm{F}$ es de 2.51, que, comparado a la $\mathrm{F}^{\prime}$ al 0.05 de margen de error, cuyo calor esperado es de 3 , no revela influencias significativas.

Al efectuar la interacción encontramos una razón $\mathrm{F}$ de 10.24 , que resulta mayor a la $\mathrm{F}^{\prime}$ al 0.05 , cuyo valor esperado es 3 , revelando que la influencia, esta vez está a favor del área de pertenencia y no del sexo.

\section{c. En la motivación del poder.}

El análisis de varianza con el grupo general arroja una razón $\mathrm{F}$ de 0.46 que resulta irrelevante.

Al contrastar los resultados en función al sexo encontramos una razón $\mathrm{F}$ de 0.53 y en función al área de pertenencia académica de 0.51; y la razón $\mathrm{F}$ de la interacción es de 2.38. Como se puede observar, en todos los casos las razones $\mathrm{F}$ calculadas resultan menores en la razón $F^{\prime}$ al 0.05 de margen de error, por lo que se concluye que ni el sexo ni el área de pertenencia tienen influencia sobre la motivación de poder.

\section{d. La motivación de logro}

Al efectuar el análisis para todo el grupo se encuentra una razón F de 0.92, no significativa. 
La contrastación en función al sexo arroja una razón $\mathrm{F}$ del 0.83 , y la correspondiente al área académica de pertenencia es de 0.1. Se puede observar que la motivación de logro no está influenciada por el sexo ni por el área académica de pertenencia.

\section{Análisis descriptivo}

Con el objeto de facilitar la descripción reproducimos las normas de interpretación de las puntuaciones directas en categorías, tanto del autoconcepto como de los componentes de la motivación que a continuación se presenta.

\section{Tabla de Conversión de Puntuaciones Directas a Categorías del Inventario de Autoconcepto}

\begin{tabular}{|c|c|}
\hline Categoría & Puntuaciones Directas \\
\hline Muy Alto & 176 hasta 200 \\
Alto & 151 hasta 175 \\
Tendencia Alto & 126 hasta 150 \\
Tendencia Bajo & 101 hasta 125 \\
Bajo & 76 hasta 100 \\
Muy Baja & 50 hasta 75 \\
\hline
\end{tabular}

Tabla de Conversión de Puntuaciones Directas a Categorías del Inventario de Motivación

\begin{tabular}{|c|c|c|c|}
\hline & \multicolumn{3}{|c|}{ Puntuaciones Directas } \\
\hline CATEGORÍA & Afiliación & Poder & Logro \\
\hline Muy Alto & $101-108$ & $101-108$ & $101-108$ \\
Alto & $83-100$ & $83-100$ & $83-100$ \\
Tendencia Alto & $65-82$ & $65-82$ & $65-82$ \\
Tendencia Bajo & $47-64$ & $47-64$ & $47-64$ \\
Bajo & $29-46$ & $29-46$ & $29-46$ \\
Muy Bajo & $18-28$ & $18-28$ & $18-28$ \\
\hline
\end{tabular}

El promedio de varones en el autoconcepto es de 144.60, que se ubica en la categoría de tendencia a alto autoconcepto. En el grupo de mujeres el promedio es de 148.48, que las ubica en similar categoría, aunque su puntaje alcanzado es ligeramente mayor al obtenido por los varones. El promedio general es de 146.31 Porcentualmente, el grupo se distribuye de la forma siguiente:

\begin{tabular}{|c|cc|}
\hline Categoría & Frecuencia & Porcentaje \\
\hline Muy Alto & 28 & $4 \%$ \\
Alto & 268 & $35 \%$ \\
Tendencia Alto & 395 & $51 \%$ \\
Tendencia Bajo & 77 & $10 \%$ \\
Bajo & - & - \\
Muy Bajo & - & - \\
& 768 & $100 \%$ \\
\hline
\end{tabular}

Como se puede observar, el $90 \%$ de los examinados presenta un autoconcepto que va de la tendencia a lato hacia muy alto, lo que deja entrever que la muestra examinada presenta un adecuado autoconcepto hacia sí mismo, hacia los demás, ante las situaciones nuevas y hacia la forma como son vistos por los demás. En la motivación de afiliación, el promedio en los 
varones es de 72.89, que corresponde a la categoría Tendencia a Alto. En las mujeres es igual a 72.82, ubicado también en la misma categoría, sin fluctuaciones significativas. En el área $\mathrm{A}$, el promedio es de 52.78, el área B de 72.11 y en el parea e es de 76.19. En todos los casos los promedios se ubican en la categoría tendencia alta, sin fluctuaciones significativas. La distribución porcentual se presenta en el cuadro correspondiente.

En la motivación de poder, el promedio de los varones es de 68.08, que corresponde a la categoría Tendencia a Alto. En las mujeres es igual a 67.32, ubicado también en la misma categoría, sin fluctuaciones significativas.

En al área A, el promedio es de 67.47, en el área B de 67.68 y en el área $\mathrm{C}$ es de 69.31. En todos los casos los promedios se ubican en la categoría tendencia alta, sin fluctuaciones significativas. La distribución porcentual se presenta en el cuadro correspondiente.

En la motivación de logro, el promedio en los varones es de 67.00, que corresponde a la categoría Tendencia Alto. En las mujeres es igual a 66.54 ubicado también en la misma categoría, sin fluctuaciones significativas.

En al área $\mathrm{A}$, el promedio es de 67.05, en el área $\mathrm{B}$ de 67.21 y en el área $\mathrm{C}$ es de 67.78. En todos los casos los promedios se ubican en la categoría tendencia alta, sin fluctuaciones significativas. La distribución porcentual se presenta en el cuadro correspondiente.

Porcentual mente, el grupo se distribuye de la forma siguiente:

\begin{tabular}{|c|cccccc|}
\hline \multirow{2}{*}{ Categoría } & \multicolumn{2}{|c}{ Afiliación } & \multicolumn{2}{c}{ Poder } & \multicolumn{2}{c|}{ Logro } \\
\cline { 2 - 7 } & Fr. & \% & Fr. & \% & Fr. & \% \\
\cline { 2 - 7 } Muy Alto & 6 & 0.78 & & & 1 & 0.39 \\
Alto & 113 & 15 & 36 & 5 & 52 & 7 \\
Tendencia Alto & 536 & 69 & 75 & 62 & 423 & 55 \\
Tendencia Bajo & 105 & 14 & 251 & 33 & 265 & 36 \\
Bajo & 8 & 1 & 6 & .78 & 9 & 1 \\
Muy Bajo & - & & - & & - & \\
\hline
\end{tabular}

Como se observa, en la afiliación, el $86 \%$ de los examinados presentan adecuada motivación de afiliación, vale decir, se encuentran orientados a satisfacer los objetivos de su grupo y se sienten realizados enmarcando sus metas con las que propone su equipo de trabajo y sus compañeros del mismo.

En la motivación de poder, el $66 \%$ encuentra en la motivación de poder un impulsor que los orienta a buscar el dominio para con el prójimo, la dirección y la centralización de la toma de decisiones y responsabilidad en sí mismo.

Por otro lado, en la motivación de logro el $63 \%$ de los evaluados alcanza un puntaje importante, lo que los califica como individuos con alto interés por sus propias tareas, encontrando la satisfacción en realizar bien las cosas y trazándose metas realistas en función a su propia capacidad individual. 


\section{DISCUSIÓN}

La ausencia de correlación entre el autoconcepto y la motivación muestra que son variables independientes, dato aparentemente contradictorio ya que el común de las investigaciones al evaluar el autoconcepto utilizan cuestionarios de autoestima, como es el caso de Coopersmhh (1967)9 encontrando influencia entre el éxito del sujeto en sus estudios y relaciones interpersonales con la autoestima, hecho que la presente investigación no es encontrado, dado que el enfoque del autoconcepto está centrado en el significado que el sujeto se atribuye, con un criterio evaluativo de su personalidad que al parecer resulta disonante con sus motivos y motivaciones.

Por tanto, el enfoque del autoconcepto, como significado del sí mismo, carente del valor que se atribuye cada quien es independiente de los componentes de la motivación de afiliación, poder y logro.

\section{CONCLUSIONES}

1. El autoconcepto y la motivación son variables independientes entre si.

2. El autoconcepto no es afectado por las variables el sexo y área de pertenencia académica.

3. La motivación de afiliación es influida por el área de pertenencia académica, siendo ligeramente mayor en el área $\mathrm{C}$.

4. La motivación de poder no es afectada por el sexo.

5. En la motivación de logros no se encuentra diferencia significativa en función al sexo ni el área de pertenencia académica.

6. El autoconcepto, en promedio y por el porcentaje hallado tiende a ser favorable en la muestra estudiada.

7. La motivación de afiliación, de poder y de logro, tanto por los promedios encontrados como por los porcentajes hallados, son buenos predictores de éxito en la culminación de sus metas. 


\section{ANEXO}

\section{DESCRIPCIÓN DE LA ESCALA DE MOTIVACIÓN}

M - L 1996

\begin{abstract}
AUTOR
COLABORADORES

AÑO
PROCEDENCIA
ADMINISTRACIÓN
OBJETIVO
\end{abstract}

TIPO DE ITIEM

ADMINISTRACIÓN

VALIDEZ
Dr. Luis Alberto Vicuna Peri

Ps. Héctor Manuel Hernández Valz

Rune Arauco Mengoni

1996

Lima - Perú

: 20 Minutos (aprox.)

: Explorar la motivación de Logro, Afiliaciòn y Poder en jóvenes y adultos. Basado en la teoría sobre las motivaciones de Mc Clelland. D.C.

Enunciados de situaciones estructuradas acompañadas por tres alternativas de respuesta obligada. Cada alternativa identifica un tipo de motivación cuyas respuestas van en una escala de seis grados que van desde el definitivamente en desacuerdo (grado 1), hasta el definitivamente de acuerdo (grado 6).

: Es necesario poner énfasis en las instrucciones de cómo debe responder el examinado, debe quedar claro que deberả contestar a cada una de las alternativas de cada situación estructurada.

La demostración si las escalas cumplen con su propósito fue establecida pot el método de análisis de contenido mediante el criterio de jueces, y por la validez de constructo.

Para la validez de contenido se puso a consideración de 10 psicólogos con experiencia en psicología de la motivación y en psicologia de las organizaciones, quienes debian opinat acerca de las situaciones estructuradas y las altemativas correspondientes a cada tipo motivacional, las que en un inicio fueron 24 quedando después del análisis solo las 18 situaciones mencionadas, las mismas que por el análisis del fi cuadrado resultaron significativas en la opinión favorable de los jueces a un nivel del 0.01 de significación.

Para el método de constructo se efectuaron correlaciones interescalas y escala total esperando encontrar correlaciones significativas entre cada uno de sus componentes y puntuaciones totales, debiendo obtenerse correlaciones significativas pero de baja intensidad con la puntuación total como un indice de que cada escala mide un componente diferente pero a su vez todas evaluan motivación encontrándose los resultados que a continuación se reproducen. 
Tabla Nro. 01 De Correlaciones Escalar, y Escalas Test Total de la Escala de Motivación

$\mathrm{M}-\mathrm{1}-96$

\begin{tabular}{lccc}
\cline { 2 - 4 } & PODER & LOGRO & TOTAL \\
\hline FULIACION & 0.72 & 0.61 & 0.42 \\
PODER & & 0.84 & 0.27 \\
LOGRO & & & 0.19 \\
\hline
\end{tabular}

CONFIABILIDAD : Fue por el método del test - retest con un intervalo de tres meses entre la primera y segunda aplicación encontrando un coeficiente de 0.88

CALIFICACıón :El computo de las puntuaciones es muy prártica, sólo debe efectuar la suma dritmética de los valores según la respuesta dada por el sujeto siguiendo la siguiente clave:

- Para fILIACIÓN sume los valores SOLO de las respuestas correspondientes a la alternativa $a$ A $\mathrm{D}$.

- Para PODER, sume los valores 50 LOO de las respuestas correspondientes a la alternativa $\circ B$.

- Para LOGRO, sume los valores a 5010 de las respuestas correspondientes a la alternativa o $\mathrm{C}$.

INTERPRETACIÓN : Las puntuaciones directas obtenida en cada una de las escalas pueden ser transformadas a categorias utilizando la siguiente tabla de conversión:

Tabla Nro. 2 De Conversión de Puntuaciones Directas a Categorias de Motivación

\begin{tabular}{cccc}
\hline CAIEGORIAS & FLLACION & PODER & LOGKOO \\
\hline Muy alto & $101-108$ & $101-108$ & $101-108$ \\
Alts & $83-100$ & $33-100$ & $83-100$ \\
Tendencia aito & $65-82$ & $65-82$ & $65-82$ \\
Tendegcia bajo & $47-64$ & $47-64$ & $47-64$ \\
Rujo & $29-46$ & $29-46$ & $29-46$ \\
Muy bajo & $18-28$ & $18-28$ & $18-28$ \\
\hline
\end{tabular}

\section{INVETIGACIÓNES EFECTUADAS CON LA ESCALA:}

a.a motivación de Logros y el autoconcepto en estudiantes universitarios de la Universidad Nacional Mayor de San Marcosx. Instituto de Investigaciones Psicológicas 1996. Dr. Luis A. Vicuña P. Hectur M. Hernández V., Jorge Luna A. Rolando Solis N., Rune Arauco M. $\checkmark$ Disposiciones Psiculógicas y Expectativas del estudiante universitario para la formación de empresas y el rol gerencialo. Instituto de Imvestigaciones Psicológicas 1997. Dr. Luis A. Vicuña P. Hector $M$. Hernández V. Mildred T. Paredes T. Rolando Solis N., Jhonan Perho G, 


\section{ESCALA M - L - 1996 \\ Por: Luis Alberto Vicuña Peri y Colaboradores}

\section{INSTRUCCIONES}

Esta escala le presenta a usted, algunas posibles situaciones. En cada una deberá indicar su grado de acuerdo o desacuerdo, para lo cual deberá poner una equis $(X)$ debajo de la categoría que describa mejor sus rasgos motivacionales. Trabaje con el siguiente criterio:

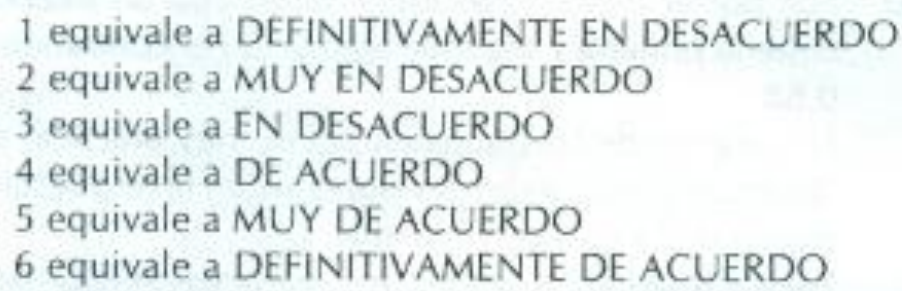

1 equivale a DEFINITIVAMENTE EN DESACUERDO

2 equivale a MUY EN DESACUERDO

3 equivale a EN DESACUERDO

4 equivale a DE ACUERDO

5 equivale a MUY DE ACUERDO

6 equivale a DEFINITIVAMENTE DE ACUERDO

\begin{tabular}{|c|c|c|c|c|c|c|}
\hline & & & acke & rto & Acuer & endo \\
\hline & & 1 & 2 & 3 & $4[5]$ & 56 \\
\hline 1. & Caumberstoy con mis padres: & & & & & \\
\hline a) & 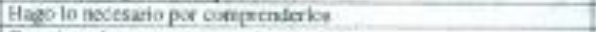 & & & & & \\
\hline b) & 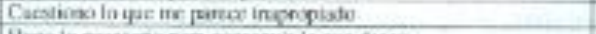 & & & & & \\
\hline c) & Hags la naxesnos pan cusequir bo que sesen & & & & & \\
\hline 2. & Ficasa: & & & & & \\
\hline D) & Apeyo en Lastomads of alyin nbytim & & & & & \\
\hline b) & Soy d yoe da foma a las bideas & & & & & \\
\hline 5) & Soy quen logra que ue lega alog anil & & & & & \\
\hline 3. & Cin mis pariuntive & & & & & \\
\hline D. & 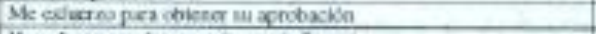 & & & & & \\
\hline b) & Hago to moresarto por evitar su iafluescia & & & & & \\
\hline (5) & 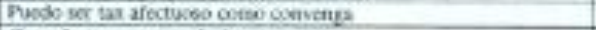 & & & & & \\
\hline 4. & Ceanbs isnge us trabaje en grupa: & & & & & \\
\hline a) & 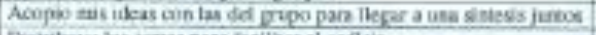 & & & & & \\
\hline b) & 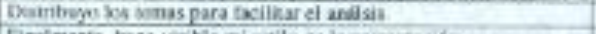 & & & & & \\
\hline d) & 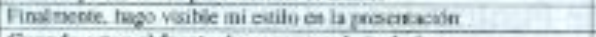 & & & & & \\
\hline 5. & Cunnde exey al fruate de un pupo de trabelon & & & & & \\
\hline a) & Mo sems al inhojo de los dembs & & & & & \\
\hline b) & Conoto el avance del tratua & & & & & \\
\hline c) & Diend pres evitar entrose & & & & & \\
\hline 6. & Si a trahaje dependiere of mi! & & & & & \\
\hline 를 & 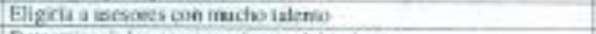 & & & & & \\
\hline b) & 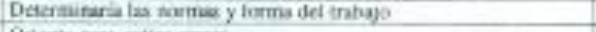 & & & & & \\
\hline 무 & Orietis porestiar chous & & & & & \\
\hline 7. & MEs anigos: & & & & & \\
\hline Q & Los irapgeriged & & & & & \\
\hline b) & Socken acia mis hitas & & & & & \\
\hline (5) & 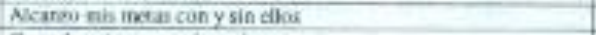 & & & & & \\
\hline K. & Cuando esiny cun mis amizies: & & & & & \\
\hline a) & Lea lovis ermin modestos & & & & & \\
\hline b) & Censim las troine que bi me juritr & & & & & \\
\hline c) & Busco la iqnoticesin de mis inciativas & & & & & \\
\hline e. & Canabs mi andige esta cen sas antipos: & & & & & \\
\hline a) & 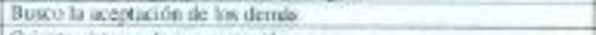 & & & & & \\
\hline b) & 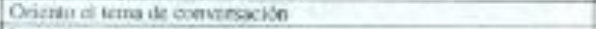 & & & & & \\
\hline c) & 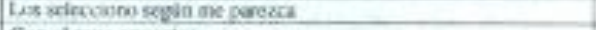 & & & & & \\
\hline 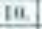 & Con el seso apuesies & & & & & \\
\hline a) & 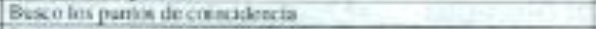 & & & & & \\
\hline b.) & Buscula forma de ocnetricar la siasciba & & & & & \\
\hline c! & Soy simpátiog si ax interese & & & & & \\
\hline 11. & D seso epuesta: & & & & & \\
\hline a) & 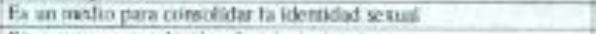 & & & & & \\
\hline b) & 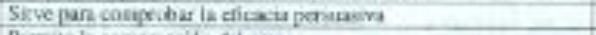 & & & & & \\
\hline D) & Permie ha congronsica del cono & & & & & \\
\hline 12. & Ea la relaciea de pareja & & & & & \\
\hline a) & 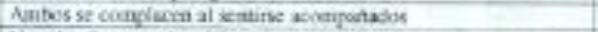 & & & & & \\
\hline D) & Len de elos ei quien debs inientar la rolasion & & & & & \\
\hline 9 & trernb por iesener mayor veilidad & & & & & \\
\hline 13. & Hexpecto a mis vecieasi & & & & & \\
\hline ai & Bexco sos lugares donde se trunen & & & & & \\
\hline b) & 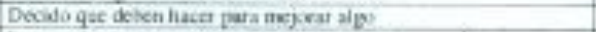 & & & & & \\
\hline c) & Les apuls sumpre que doverge un benchicis & & & & & \\
\hline
\end{tabular}




\section{REFERENCIAS}

1. Ellis, A. Razón y Emoción en Psicoterapia. Ed. Descle de Browner, España. 1994

2. Mahoney, M. Congnición y Modificación de Conducta. Ed. Trillas, México 1988

3. Kuch Joanne. Aprendiendo a Conocerme. Ed. Globe Booak Company, 1994

4. Madsen, K.B. «Teorías de la Motivación». Buenos Aires. Paidos 1977

5. Mc Clelland D.e. «Studies in Motivation». N.Y. appleton century 1953

6. Peyró, José M. Psicología de las Organizaciones, Tomo 11. Biblioteca Nacional de Aprendizaje a Distancia.1984

7. Brown, Rogers.»Psicología Social». Siglo XXI editores. Buenos Aires Argentina 1974

8. Brown, Rogers.»Motivación y Actitudes».Siglo XXI editores. Buenos Aires Argentina 1974

9. Coopersmith S. The antecedents of selfsteem. Palo Alto Consulting Psichologists Press. USA. 1967 